\title{
Effects of compound danshen dripping pills on the levels of serum inflammatory factors, brain natriuretic peptide and blood lipid in CABG postoperative patients
}

\author{
Guizhu Zhao ${ }^{1}$, Dongmei Zhang ${ }^{2}$, Zhanjun Sun ${ }^{1}$, Xia Yang*1 \\ ${ }^{1}$ Baogang Hospital, Baotou, Inner Mongolia, China \\ ${ }^{2}$ Baotou Food and Drug Inspection Center, Baotou, Inner Mongolia, China
}

Received: July 30, 2020

DOI: $10.5430 /$ dcc.v7n2p13
Accepted: October 15, 2020

Online Published: November 10, 2020

\begin{abstract}
Objective: To explore the effects of compound danshen dripping pills on the levels of serum inflammatory factors, brain natriuretic peptide (BNP) and blood lipid in CABG postoperative patients.

Methods: 156 cases of patients who were given CABG from January 2015 to January 2018 in Baogang Hospital were chosen and randomly divided into the observation group $(\mathrm{n}=78)$ and the control group $(\mathrm{n}=78)$. Both groups were given conventional drugs, simultaneously, the observation group was given compound danshen dripping pills. The treatment lasted 3 months. The clinical efficacy was compared between two groups of patients, and the levels of serum inflammatory factors, plasma BNP and blood lipid before and after treatment in two groups of patients were detected in order to make a comparison in the incidence of adverse cardiac events between two groups of patients.

Results: After treatment, the total effective rate of treatment in the observation group was significantly higher than that in the control group; in the two groups, the levels of serum interleukin-6 (IL-6), interleukin-8 (IL-8), C-reactive protein and plasma BNP after treatment were obviously lower than those before treatment, and these indicators in the observation group were lower than those in the control group $(p<.05)$; the levels of TG, TC and LDL-C in two groups were obviously lower $(p<.05)$ with the level of HDL-C significantly higher (all $p<.05$ ), and the descending or ascending range of each indicator in the observation group was obviously larger than that in the control group $(p<.05)$; in the follow-up visit, the incidence of adverse cardiac events in the observation group of patients was significantly lower than that in the control group of patients $(p<.05)$.

Conclusions: Compound danshen dripping pills can effectively reduce the levels of serum inflammatory factors and BNP, regulate blood lipid metabolism and reduce the incidence of adverse cardiac events in CABG postoperative patients.
\end{abstract}

Key Words: CABG, Compound danshen dripping pills, Inflammatory factors, Brain natriuretic peptide, Blood lipid

\section{INTRODUCTION}

Coronary artery bypass grafting $(\mathrm{CABG})$ is internationally considered as the most effective way to treat severe CHD, and it has been applied clinically for more than 30 years. The operation can quickly relieve angina pectoris and other symptoms by bridging aorta or the distal portion of the obstructed

*Correspondence: Xia Yang; Email: yangxia00426@126.com; Address: Baogang Hospital, Baotou, Inner Mongolia 014010, China. 
coronary artery to the autograft blood vessel and building up a new vascular access to improve cardiac blood supply; ${ }^{[1]}$ however, if there is no effective bypass protection treatment after surgery, patients with CHD are still endangered with secondary vascular obstruction, which is not beneficial to the prognosis and even threatens life and health. ${ }^{[2]}$ Some researches have indicated that compound danshen dripping pills can promote blood circulation and remove blood stasis, regulate qi and relieve pain, improve myocardial blood supply and the microcirculation. ${ }^{[3]}$ This research was designed to explore the application value of compound danshen dripping pills to $\mathrm{CABG}$ postoperative patients and provide a reference to the clinical treatment.

\section{Clinical data}

\subsection{General information}

156 cases of patients who were given CABG from January 2015 to January 2018 in Baogang Hospital were chosen, and the diagnostic criteria of western medicine was based on the related diagnostic standards for cardiovascular and cerebrovascular diseases established by WHO; ${ }^{[4]}$ the diagnostic criteria of traditional Chinese medicine conformed to the diagnostic standards illustrated in "Chinese Medicine Diagnostics of Cardia-cerebrovascular Disease"; ${ }^{5]}$ during the process of diagnosis, it was required to give sufficient consideration to clinical symptoms and imageological examination data. Inclusion criteria: patients who conformed to the abovementioned diagnostic standards and were diagnosed as CHD according to the imageological examinations; patients who conformed to the indications for CABG and successfully went through the operation; patients with different degrees of dyspnea, tachypnea or edema etc.; as to the patients with unstable angina, the degree of disease was required to be controlled and kept stable for at least one week. Exclusion criteria: patients with hepatic or renal dysfunction; patients with malignant tumors; patients with systemic or local infection; patients who was allergic to the research drug; patients who received other treatments. Withdrawal and elimination criteria: patients who had severe adverse events during the treatment; patients who failed to take medicine under medical supervision; patients who lost contact during the process of treatment or observation. All patients were randomly divided into the observation group $(n=78)$ and the control group $(\mathrm{n}=78)$. In the observation group, there were 44 cases of male patients and 34 cases of female patients; aged 57 to $76(63.7 \pm 6.1)$; BMI $(21.10 \pm 3.65) \mathrm{kg} / \mathrm{m}^{2}$; disease type: 24 cases of myocardial infarction, 37 cases of unstable angina pectoris, 14 cases of cardiac failure and 3 cases of others; classification of NYHA heart function after operation: 40 cases of NYHA Grade II and 38 cases of NYHA
Grade III. In the observation group, there were 47 cases of male patients and 31 cases of female patients; aged 58 to 78 $(64.4 \pm 6.2)$; BMI $(21.47 \pm 3.71) \mathrm{kg} / \mathrm{m}^{2}$; disease type: 27 cases of myocardial infarction, 34 cases of unstable angina pectoris, 13 cases of cardiac failure and 4 cases of others; classification of NYHA heart function after operation: 45 cases of NYHA Grade II and 33 cases of NYHA Grade III. According to the statistical analysis, the differences were of no statistical significance in general data such as age, gender, BMI, disease type and classification of NYHA heart function of two groups of patients $(p>.05)$, and the comparative study was applicable to the comparison between two groups.

\subsection{Treatment methods}

Two groups of patients were given unified diet, and it was required to guarantee the intake of fresh fruit and vegetables and nutrient substances. After surgery, the control group of patients were given conventional drugs, including nitrates, statins, aspirin, ACE inhibitors and so on. On the basis of the control group, the observation group of patients were given compound danshen dripping pills additionally (TASLY PHARMACEUTICAL GROUP CO., LTD., SFDA Approval No.: Z10950111, 180 pills/bottle), 10 pills/time, 3 times/d. The two groups were given 3 -month continuous treatment.

\subsection{Indicator observation}

1) The statistical data of the clinical efficacy after treatment in two groups were collected in order to evaluate the clinical efficacy in two groups of patients according to "Guidelines for the clinical study of new traditional Chinese medicine". [6] 2) Before and after treatment, $5 \mathrm{ml}$ of fasting peripheral venous blood was taken from each patient in two groups in the morning, and the supernatant was taken after centrifugation. ELISA was used to detect the levels of serum inflammatory factors and blood lipid, including interleukin-6 (IL-6), interleukin-8 (IL-8), C-reactive protein (CRP), triglyceride (TG), total cholesterol (TC), high density lipoprotein cholesterol (HDL-C) and low density lipoprotein cholesterol (LDLC). 3) The immunochemiluminometric assays were applied to the detection of BNP level. 4) The one-year follow-up visit was made towards all patients in order to calculate the incidence of adverse cardiac events in two groups of patients.

\subsection{Judgment standards for the clinical efficacy}

Cured: the clinical symptoms disappeared almost, with normal examination results, the cardiac function recovered to NYHA Grade I; improving: the clinical symptoms and the examination results were improved, the cardiac function was improved to Grade 2 but didn't reach up to NYHA Grade I; effective: the clinical symptoms and the examination results were improved, the cardiac function was improved to Grade 
1 but didn't reach up to NYHA Grade I; ineffective: the above-mentioned symptoms were not improved, the patients couldn't participate in the regular activities and work, with recurrent seizures during the follow-up visit. The total effective rate $=$ the number of $($ Effective + Improving + Cured $)$ patients/the total number of patients $\times 100 \%$.

\subsection{Statistical methods}

SPSS 20.0 software was applied to the analysis of the above data. The categorical data and the measurement data were represented by $\%$ and $\bar{X} \pm$ s respectively, with $t$-test and $\chi^{2}$ used in the comparison between two groups. The difference $p<.05$ was of statistical significance.

\section{RESUlts}

\subsection{The comparison in the clinical efficacy between two groups}

After treatment, the total effective rate of the observation group was higher than that of the control group $(p<.05)$.
See Table 1 for details.

\subsection{The comparison in the levels of BNP and inflamma- tory factors between two groups}

After treatment, the levels of CRP, IL-6, IL-8 in the serum and BNP in the plasma in the observation group were significantly lower than those in the control group $(p<.05)$. See Table 2 for details.

\subsection{The comparison in the level of blood lipid between two groups}

After treatment, the levels of serum TC, TG and LDL-C were lower in the two groups, and these indicators in the observation group were lower than those in the control group; the level of HDL-C was higher in the two groups, and it was much higher in the observation group. The difference was statistically significant $(p<.05)$. See Table 3 for details.

Table 1. The comparison in the clinical efficacy between two groups of CABG postoperative patients $\mathrm{n}(\%)$

\begin{tabular}{lllllll}
\hline Group & $\mathbf{n}$ & Cured & Improving & Effective & Ineffective & Total Effective Rate \\
\hline Observation Group & 78 & $38(48.72)$ & $21(26.92)$ & $16(20.51)$ & $3(3.85)$ & $75(96.15)$ \\
Control Group & 78 & $17(21.79)$ & $25(32.05)$ & $22(28.21)$ & $14(17.95)$ & $64(82.05)$ \\
\hline
\end{tabular}

Note. ${ }^{*}$ In Comparison with the control group, $p<.05$

Table 2. The comparison in the levels of BNP and inflammatory factors between two groups of CABG postoperative patients $(\bar{X} \pm \mathrm{s})$

\begin{tabular}{|c|c|c|c|c|c|c|}
\hline Group & n & Time & $\mathrm{BNP} /(\mathrm{pg} / \mathrm{ml})$ & CRP/(mg/L) & IL-6/(pg/ml) & IL-8/(pg/ml) \\
\hline Observation & 78 & Before Treatment & $854.12 \pm 69.73$ & $27.98 \pm 2.95$ & $36.83 \pm 7.25$ & $8.94 \pm 1.35$ \\
\hline Group & 10 & After Treatment & $363.58 \pm 36.24^{* \#}$ & $14.46 \pm 0.81$ *\# & $20.11 \pm 5.27^{* \#}$ & $5.20 \pm 1.24 * \#$ \\
\hline Control & 78 & Before Treatment & $858.09 \pm 68.72$ & $28.20 \pm 2.96$ & $36.85 \pm 7.32$ & $9.01 \pm 1.17$ \\
\hline Group & 10 & After Treatment & $602.17 \pm 45.69^{*}$ & $21.64 \pm 2.17^{*}$ & $26.64 \pm 4.39^{*}$ & $7.15 \pm 0.92^{*}$ \\
\hline
\end{tabular}

Note. ${ }^{*}$ In comparison with the indicators before treatment, $p<.05 ;{ }^{*}$ in comparison with the control group, $p<.05$.

Table 3. The comparison in the level of blood lipid between two groups of CABG postoperative patients $(\bar{X} \pm \mathrm{s}, \mathrm{mmol} / \mathrm{L})$

\begin{tabular}{lllllll}
\hline Group & $\mathbf{n}$ & Time & TG & TC & HDL-C & LDL-C \\
\hline \multirow{2}{*}{ Observation Group } & \multirow{2}{*}{78} & Before Treatment & $2.33 \pm 0.56$ & $6.31 \pm 2.25$ & $1.09 \pm 0.34$ & $3.56 \pm 0.64$ \\
& & After Treatment & $1.74 \pm 0.57^{* \#}$ & $4.29 \pm 1.71^{* \#}$ & $1.41 \pm 0.42^{* \#}$ & $2.61 \pm 0.60{ }^{* \#}$ \\
\multirow{2}{*}{ Control Group } & \multirow{2}{*}{78} & Before Treatment & $2.36 \pm 0.54$ & $6.27 \pm 2.34$ & $1.07 \pm 0.40$ & $3.58 \pm 0.65$ \\
& & After Treatment & $2.12 \pm 0.60^{*}$ & $5.22 \pm 1.83^{*}$ & $1.26 \pm 0.49^{*}$ & $3.01 \pm 0.44^{*}$ \\
\hline
\end{tabular}

Note. ${ }^{*}$ In comparison with the indicators before treatment, $p<.05 ;{ }^{\#}$ in comparison with the control group, $p<.05$.

Table 4. The comparison in the incidence of adverse cardiac events between two groups of CABG postoperative patients $\mathrm{n}(\%)$

\begin{tabular}{llllll}
\hline Group & $\mathbf{n}$ & Malignant Arrhythmia & Myocardial Infarction & Deterioration of Cardiac Failure & Total \\
\hline Observation Group & 78 & $2(2.56)$ & $1(1.28)$ & $1(1.28)$ & $4(5.12)^{*}$ \\
Control Group & 78 & $7(8.97)$ & $3(3.85)$ & $10(12.82)$ & $20(25.64)$ \\
\hline
\end{tabular}

Note. ${ }^{*}$ In comparison with the control group, $p<.05$ 


\subsection{The incidence of adverse cardiac events in two groups}

In the follow-up visit, the incidence of adverse cardiac events in the observation group was lower than that in the control group $(p<.05)$. See Table 4 for details.

\section{Discussion}

CABG has become an effective way to treat CHD. This operation is designated to improve myocardial ischemia and relieve angina pectoris by transplanting autogenous vessels, in order to reduce the morality and improve the quality of life. ${ }^{[7,8]}$ However, as patients with CHD are accompanied with different degrees of symptoms such as cardiac trauma and cardiac failure, they are still potentially endangered with coronarospasm, myocardial infarction, abnormal flow and so on after CABG. In addition, surgical injury can also lead to cardiac failure or aggravate preexisting cardiac failure. ${ }^{[9]}$ Therefore, how to effectively prevent and treat cardiac failure after surgery subsequently becomes a clinical research focus.

From the perspective of traditional Chinese medicine, it is considered that the medicine which can supplement Qi to warm Yang should be applied to the treatment of CHD, with the supplement of drugs which can promote blood circulation to remove blood stasis and dredge collaterals to alleviate edema. ${ }^{[10,11]}$ This perspective is consistent with the modern therapeutic concept: the key to the treatment of CHD is to reduce cardiac preload and afterload while increasing cardiac output and relieve clinical symptoms while restoring cardiac function. ${ }^{[12]}$ Compound danshen dripping pills are made up of radix salviae miltiorrhizae, borneol, panax notoginseng and so on, and this medicine can promote blood circulation to remove blood stasis and activate collaterals to alleviate pains. According to modern pharmacology experiments, it is confirmed that compound danshen dripping pills can inhibit serum inflammatory factors, stabilize atheromatous plaques, improve blood lipid and hemodynamics and depress ventricular remodeling in order to effectively protect the heart and myocardial cells. ${ }^{[13]}$ The research results have shown that the total effective rate after treatment in the observation group is significantly higher than that in the control group, which conforms to the research results from HONG Jiankang et al. ${ }^{[14]}$ It is indicated that compound danshen dripping pills can effectively improve the condition of myocardial blood supply in patients with $\mathrm{CABG}$, and it is probably related to its effects on dilating coronary vessels, lowering vascular resistance and improving myocardial ischemia and oxygen consumption.

A large number of clinical researches have indicated that inflammatory factors play an important role in the occur- rence and development of CHD. CRP, which is an indicator of inflammatory responses, can be used to evaluate the cardiac function and the risk in postoperative patients; IL-6 and IL-8, which are important regulatory factors of inflammatory responses, participate in the process of myocardial damage mediated by neutrophils. ${ }^{[15]}$ In this research, the results have shown that the levels of IL-6, IL-8 and CRP after treatment in the observation group are lower than those in the control group, which is consistent with the research results from Fang Fang et al. ${ }^{[16]}$ It is indicated that compound danshen dripping pills can restrain atherosclerotic plaques from releasing inflammatory factors in order to protect vascular smooth muscle, suppress platelet aggregation and prevent blood clotting. BNP is a type of polypeptide hormone which is synthesized and secreted by ventriculus sinister. The content of BNP is significantly increased after myocardial infarction or cardiac failure happen to patients. It can be used as an important indicator in the evaluation of the prognosis in CABG postoperative patients. ${ }^{[17]}$ In the research conducted by Xiang Zhang et al., ${ }^{[18]}$ it is found that the level of BNP in CHD patients is closely associated with the type and the severity of CHD. BNP level can accurately reflect the degree of CHD. In this research, it is found that the levels of BNP in two groups of patients are lower after treatment, and the level of BNP in the observation group is lower than that in the control group. The results are similar to the research results from Yongqiang Liu et al. ${ }^{[19]}$ It is indicated that the application of compound danshen dripping pills can effectively reduce the level of BNP and improve the prognosis in CABG postoperative patients with CHD. It is also confirmed that the incidence of adverse cardiac events in the observation group is significantly lower than that in the control group during the process of follow-up visit. Currently, it has been confirmed that blood lipid metabolism is closely associated with the occurrence of CHD, and it has been generally considered as one of risk factors for the onset of CHD. In this research, the improvement of blood lipid in the observation group is superior to the control group. It is indicated that the application of compound danshen dripping pills contributes to the regulation of blood lipid metabolism in $\mathrm{CABG}$ postoperative patients.

In conclusion, the application of compound danshen dripping pills can effectively reduce the levels of serum inflammatory factors and BNP, contribute to the regulation of blood lipid metabolism and reduce the incidence of adverse cardiac events in order to improve the prognosis with a significant, safe and reliable clinical efficacy.

\section{CONFLICTS OF INTEREST DISCLOSURE}

The authors declare they have no conflicts of interest. 


\section{REFERENCES}

[1] Shoufeng Du. Anesthesia management and therapeutic effect of offpump CABG patients with CHD accompanying diabetes. Chinese Journal of Gerontology. 2016; 36(3): 666-668.

[2] Lv J, Wang Z. Research progress on the prevention and treatment of myocardial ischemia reperfusion injury with traditional Chinese medicine. Jiangsu Journal of Traditional Chinese Medicine. 2012; 44(5): 76-77.

[3] Mack MJ, Head SJ, Jr HD, et al. Analysis of stroke occurring in the SYNTAX trial comparing coronary artery bypass surgery and percutaneous coronary intervention in the treatment of complex coronary artery disease. Jacc Cardiovascular Interventions. 2013; 6(4): 344-354.

[4] Liang F, Shen Z. Present state and perspectives of the diagnosis and treatment of CHD with acute cardiac failure. Journal of Clinical Internal Medicine. 2016; 33(1): 16-20.

[5] Guo Q. Chinese Medicine Diagnostics of Cardia-cerebrovascular Disease. Beijing: TCM Classics Publishing House; 2013.

[6] National Administration of Traditional Chinese Medicine. Guidelines for the clinical study of new traditional Chinese medicine (Trial). Beijing: China Medical Science Press; 2002.

[7] Lei B. Discussion on the clinical effect and long-term efficacy of coronary artery bypass grafting $[\mathrm{J} / \mathrm{CD}]$. Cardiovascular Disease Journal Of integrated traditional Chinese and Western Medicine (Electronic Edition). 2016; 4(25): 63-64.

[8] Ren A. Analysis on the effect of trimetazidine with compound Danshen preparation on CABG postoperative patients with cardiac failure. Chinese Journal of Practical Medicine. 2016; 43(15): 73-74.

[9] Chen C, Li X, Chen Y, et al. Observation and nursing of CABG complications with CHD accompanying diabetes. Zhejiang Clinical Medical Journal. 2015; 17(9): 1640-1642.

[10] Xiao M, Liu Y, Hao J, et al. Understanding and case analysis on the treatment of cardiac failure in traditional Chinese medicine. World Chinese Medicine. 2011; 6(6): 495-496.
[11] Feng S, Teng G, Qu Y. Clinical analysis on clinical pathway optimization program of the treatment of cardiac failure with favored originpreserving decoction. Guangming Journal of Chinese Medicine. 2015; 30(2): 288-289.

[12] Qiu Y, Liu T, Tang S, et al. Pharmaceutical care for patients with chronic heart failure. Chinese Journal of Hospital Pharmacy. 2013; 33(11): 914-916.

[13] Chen Y. Analysis on the application and pharmacological action of Compound Danshen dropping pills in cardiovascular diseases. Medical Frontier. 2016; 6(35): 336-337.

[14] Hong J, Hou X, Xiao X, et al. Effect of compound Danshen dropping Pills on B-type brain natriuretic peptide and C-reactive protein in patients with chronic heart failure with type 2 diabetes mellitus. Chinese Medical Science. 2013; 3(20): 49-50.

[15] Lu H, Li M, Wang Y, et al. The value of 5 inflammatory markers in the determination of atherosclerosis and the risk of coronary heart disease. Journal of Practical Medicine. 2016; 32(2): 203-207.

[16] Fang F, Gan S, Feng G, et al. Effects of long-term administration of Compound Danshen dripping Pills on left ventricular remodeling and inflammatory factor levels in elderly patients with acute myocardial infarction after PCI. Advances in Modern Biomedicine. 2017; 17(3): 544-546.

[17] Guo Y. Effect of compound Danshen dropping pills on cardiac function and BNP level in patients with chronic heart failure. Journal of Integrated Chinese and Western Medicine cardio-Cerebrovascular Disease. 2013; 11(12): 1444-1445.

[18] Zhang X, Liu F, Chen X. Levels of brain natriuretic peptide, peptide hormone and $\mathrm{C}$-reactive protein in patients with different types of coronary heart disease. Journal of Hainan Medical College. 2013; 19(12): 1653-1655.

[19] Liu Y, Fu Q, Han P, et al. Effect of compound Danshen preparation and trimetazidine on BNP and CRP in patients with heart failure after coronary artery bypass grafting. Modern Preventive Medicine. 2012; 39(8): 2001-2002. 